\title{
Existence of solutions and convergence analysis for a system of quasivariational inclusions in Banach spaces
}

\author{
Jia-wei Chen ${ }^{1,2^{*}}$ and Zhongping Wan ${ }^{1}$
}

\author{
* Correspondence: J.W. \\ Chen713@163.com \\ ${ }^{1}$ School of Mathematics and \\ Statistics, Wuhan University, \\ Wuhan, Hubei 430072, PR China \\ Full list of author information is \\ available at the end of the article
}

\begin{abstract}
In order to unify some variational inequality problems, in this paper, a new system of generalized quasivariational inclusion (for short, (SGQVI)) is introduced. By using Banach contraction principle, some existence and uniqueness theorems of solutions for (SGQVI) are obtained in real Banach spaces. Two new iterative algorithms to find the common element of the solutions set for (SGQVI) and the fixed points set for Lipschitz mappings are proposed. Convergence theorems of these iterative algorithms are established under suitable conditions. Further, convergence rates of the convergence sequences are also proved in real Banach spaces. The main results in this paper extend and improve the corresponding results in the current literature. 2000 MSC: 47H04; 49J40.
\end{abstract}

Keywords: system of generalized quasivariational inclusions problem, strong convergence theorem, convergence rate, resolvent operator, relaxed cocoercive mapping

\section{Introduction}

Variational inclusion problems, which are generalizations of variational inequalities introduced by Stampacchia [1] in the early sixties, are among the most interesting and intensively studied classes of mathematics problems and have wide applications in the fields of optimization and control, economics, electrical networks, game theory, engineering science, and transportation equilibria. For the past decades, many existence results and iterative algorithms for variational inequality and variational inclusion problems have been studied (see, for example, [2-13]) and the references cited therein). Recently, some new and interesting problems, which are called to be system of variational inequality problems, were introduced and investigated. Verma [6], and Kim and Kim [7] considered a system of nonlinear variational inequalities, and Pang [14] showed that the traffic equilibrium problem, the spatial equilibrium problem, the Nash equilibrium, and the general equilibrium programming problem can be modeled as a system of variational inequalities. Ansari et al. [2] considered a system of vector variational inequalities and obtained its existence results. Cho et al. [8] introduced and studied a new system of nonlinear variational inequalities in Hilbert spaces. Moreover, they obtained the existence and uniqueness properties of solutions for the system of nonlinear variational inequalities. Peng and Zhu [9] introduced a new system of generalized mixed quasivariational inclusions involving $(H, \eta)$-monotone operators. Very

(c) 2011 Chen and Wan; licensee Springer. This is an Open Access article distributed under the terms of the Creative Commons Attribution License (http://creativecommons.org/licenses/by/2.0), which permits unrestricted use, distribution, and reproduction in any medium, provided the original work is properly cited. 
recently, Qin et al. [15] studied the approximation of solutions to a system of variational inclusions in Banach spaces and established a strong convergence theorem in uniformly convex and 2-uniformly smooth Banach spaces. Kamraksa and Wangkeeree [16] introduced a general iterative method for a general system of variational inclusions and proved a strong convergence theorem in strictly convex and 2-uniformly smooth Banach spaces. Wangkeeree and Kamraksa [17] introduced an iterative algorithm for finding a common element of the set of solutions of a mixed equilibrium problem, the set of fixed points of an infinite family of nonexpansive mappings, and the set of solutions of a general system of variational inequalities, and then proved the strong convergence of the iterative in Hilbert spaces. Petrot [18] applied the resolvent operator technique to find the common solutions for a generalized system of relaxed cocoercive mixed variational inequality problems and fixed point problems for Lipschitz mappings in Hilbert spaces. Zhao et al. [19] obtained some existence results for a system of variational inequalities by Brouwer fixed point theory and proved the convergence of an iterative algorithm infinite Euclidean spaces.

Inspired and motivated by the works mentioned above, the purpose of this paper is to introduce and investigate a new system of generalized quasivariational inclusions (for short, (SGQVI)) in q-uniformly smooth Banach spaces, and then establish the existence and uniqueness theorems of solutions for the problem (SGQVI) by using Banach contraction principle. We also propose two iterative algorithms to find the common element of the solutions set for (SGQVI) and the fixed points set for Lipschitz mappings. Convergence theorems with estimates of convergence rates are established under suitable conditions. The results presented in this paper unifies, generalizes, and improves some results of [6,15-20].

\section{Preliminaries}

Throughout this paper, without other specifications, we denote by $Z_{+}$and $R$ the set of non-negative integers and real numbers, respectively. Let $E$ be a real $q$-uniformly Banach space with its dual $\left.E^{*}, q\right\rangle 1$, denote the duality between $E$ and $E^{*}$ by $\langle\cdot, \cdot\rangle$ and the norm of $E$ by $\|\cdot\|$, and $T: E \rightarrow E$ be a nonlinear mapping. When $\left\{x_{n}\right\}$ is a sequence in $E$, we denote strong convergence of $\left\{x_{n}\right\}$ to $x \in E$ by $x_{n} \rightarrow x$. A Banach space $E$ is said to be smooth if $\lim _{t \rightarrow 0} \frac{\|x+t y\|-\|x\|}{t}$ exists for all $x, y \in E$ with $\|x\|=\|y\|$ $=1$. It is said to be uniformly smooth if the limit is attained uniformly for $\|x\|=\|y\|$ $=1$. The function

$$
\rho_{E}(t)=\sup \left\{\frac{\|x+y\|+\|x-y\|}{2}-1:\|x\|=1,\|y\| \leq t\right\}
$$

is called the modulus of smoothness of $E$. $E$ is called $q$-uniformly smooth if there exists a constant $c>0$ such that $\rho_{E}(t) \leq c t^{q}$.

Example 2.1.[20] All Hilbert spaces, $L^{p}\left(\right.$ or $\left.l^{p}\right)$ and the Sobolev spaces $W_{m}^{p},(p \geq 2)$ are 2-uniformly smooth, while $L^{p}$ (or $\left.l^{p}\right)$ and $W_{m}^{p}$ spaces $(1<p \leq 2)$ are $p$-uniformly smooth. The generalized duality mapping $J_{q}: E \rightarrow 2 E^{*}$ is defined as

$$
J_{q}(x)=\left\{f^{*} \in E^{*}:\left\langle f^{*}, x\right\rangle=\left\|f^{*}\left|\||| x||=\| x\left\|^{q},\right\| f^{*}\|=\| x \|^{q-1}\right\}\right.\right.
$$

for all $x \in E$. Particularly, $J=J_{2}$ is the usual normalized duality mapping. It is wellknown that $J_{q}(x)=\|x\|^{q-2} J(x)$ for $x \neq 0, J_{q}(t x)=t^{q-1} J_{q}(x)$, and $J_{q}(-x)=-J_{q}(x)$ for all $x \in$ 
$E$ and $t \in[0,+\infty)$, and $J_{q}$ is single-valued if $E$ is smooth. If $E$ is a Hilbert space, then $J$ $=I$, where $I$ is the identity mapping. Many properties of the normalized duality mapping $J_{q}$ can be found in (see, for example, [21]). Let $\rho_{1}, \rho_{2}$ be two positive constants, $A_{1}, A_{2}: E \times E \rightarrow E$ be two single-valued mappings, $M_{1}, M_{2}: E \rightarrow 2^{E}$ be two set-valued mappings. The (SGQVI) problem is to find $\left(x^{*}, y^{*}\right) \in E \times E$ such that

$$
\left\{\begin{array}{l}
0 \in x^{*}-y^{*}+\rho_{1}\left(A_{1}\left(y^{*}, x^{*}\right)+M_{1}\left(x^{*}\right)\right), \\
0 \in y^{*}-x^{*}+\rho_{2}\left(A_{2}\left(x^{*}, y^{*}\right)+M_{2}\left(y^{*}\right)\right) .
\end{array}\right.
$$

The set of solutions to (SGQVI) is denoted by $\Omega$.

Special examples are as follows:

(I) If $A_{1}=A_{2}=A, E=H$ is a Hilbert space, and $M_{1}(x)=M_{2}(x)=\partial \varphi(x)$ for all $x \in$ $E$, where $\varphi: E \rightarrow R \cup\{+\infty\}$ is a proper, convex, and lower semicontinuous functional, and $\partial \varphi$ denotes the subdifferential operator of $\varphi$, then the problem (SGQVI) is equivalent to find $\left(x^{*}, y^{*}\right) \in E \times E$ such that

$$
\left\{\begin{array}{l}
\left\langle\rho_{1} A\left(y^{*}, x^{*}\right)+x^{*}-y^{*}, x-x^{*}\right\rangle+\phi(x)-\phi\left(x^{*}\right) \geq 0, \forall x \in E, \\
\left\langle\rho_{2} A\left(x^{*}, y^{*}\right)+y^{*}-x^{*}, x-y^{*}\right\rangle+\phi(x)-\phi\left(y^{*}\right) \geq 0, \forall x \in E,
\end{array}\right.
$$

where $\rho_{1}, \rho_{2}$ are two positive constants, which is called the generalized system of relaxed cocoercive mixed variational inequality problem [22].

(II) If $A_{1}=A_{2}=A, E=H$ is a Hilbert space, and $K$ is a closed convex subset of $E$, $M_{1}(x)=M_{2}(x)=\partial \varphi(x)$ and $\varphi(x)=\delta_{K}(x)$ for all $x \in E$, where $\delta_{K}$ is the indicator function of $K$ defined by

$$
\phi(x)=\delta_{K}(x)= \begin{cases}0 & \text { if } x \in K, \\ +\infty & \text { otherwise },\end{cases}
$$

then the problem (SGQVI) is equivalent to find $\left(x^{*}, y^{*}\right) \in K \times K$ such that

$$
\left\{\begin{array}{l}
\left\langle\rho_{1} A\left(y^{*}, x^{*}\right)+x^{*}-y^{*}, x-x^{*}\right\rangle \geq 0, \forall x \in K, \\
\left\langle\rho_{2} A\left(x^{*}, y^{*}\right)+y^{*}-x^{*}, x-y^{*}\right\rangle \geq 0, \forall x \in K,
\end{array}\right.
$$

where $\rho_{1}, \rho_{2}$ are two positive constants, which is called the generalized system of relaxed cocoercive variational inequality problem [23].

(III) If for each $i \in\{1,2\}, z \in E, A_{i}(x, z)=\Psi_{i}(x)$, for all $x \in E$, where $\Psi_{i}: E \rightarrow E$, then the problem (SGQVI) is equivalent to find $\left(x^{*}, y^{*}\right) \in E \times E$ such that

$$
\left\{\begin{array}{l}
0 \in x^{*}-y^{*}+\rho_{1}\left(\Psi_{1}\left(y^{*}\right)+M_{1}\left(x^{*}\right)\right), \\
0 \in y^{*}-x^{*}+\rho_{2}\left(\Psi_{2}\left(x^{*}\right)+M_{2}\left(y^{*}\right)\right),
\end{array}\right.
$$

where $\rho_{1}, \rho_{2}$ are two positive constants, which is called the system of quasivariational inclusion $[15,16]$.

(IV) If $A_{1}=A_{2}=A$ and $M_{1}=M_{2}=M$ then the problem (SGQVI) is reduced to the following problem: find $\left(x^{*}, y^{*}\right) \in E \times E$ such that

$$
\left\{\begin{array}{l}
0 \in x^{*}-y^{*}+\rho_{1}\left(A\left(y^{*}, x^{*}\right)+M\left(x^{*}\right)\right), \\
0 \in y^{*}-x^{*}+\rho_{2}\left(A\left(x^{*}, y^{*}\right)+M\left(y^{*}\right)\right),
\end{array}\right.
$$

where $\rho_{1}, \rho_{2}$ are two positive constants.

(V) If for each $i \in\{1,2\}, z \in E, A_{i}(x, z)=\Psi(x)$, and $M_{1}(x)=M_{2}(x)=M$, for all $x \in$ $E$, where $\Psi: E \rightarrow E$, then the problem (SGQVI) is equivalent to find $\left(x^{*}, y^{*}\right) \in E \times E$ such that 


$$
\left\{\begin{array}{l}
0 \in x^{*}-y^{*}+\rho_{1}\left(\Psi\left(y^{*}\right)+M\left(x^{*}\right)\right) \\
0 \in y^{*}-x^{*}+\rho_{2}\left(\Psi\left(x^{*}\right)+M\left(y^{*}\right)\right)
\end{array}\right.
$$

where $\rho_{1}, \rho_{2}$ are two positive constants, which is called the system of quasivariational inclusion [16].

We first recall some definitions and lemmas that are needed in the main results of this work.

Definition 2.1.[21] Let $M: \operatorname{dom}(M) \subset E \rightarrow 2^{E}$ be a set-valued mapping, where dom $(M)$ is effective domain of the mapping $M . M$ is said to be

(i) accretive if, for any $x, y \in \operatorname{dom}(M), u \in M(x)$ and $v \in M(y)$, there exists $j_{q}(x-y)$ $\in J_{q}(x-y)$ such that

$$
\left\langle u-v, j_{q}(x-\gamma)\right\rangle \geq 0 .
$$

(ii) $m$-accretive (maximal-accretive) if $M$ is accretive and $(I+\rho M) \operatorname{dom}(M)=E$ holds for every $\rho>0$, where $I$ is the identity operator on $E$.

Remark 2.1. If $E$ is a Hilbert space, then accretive operator and $m$-accretive operator are reduced to monotone operator and maximal monotone operator, respectively.

Definition 2.2. Let $T: E \rightarrow E$ be a single-valued mapping. $T$ is said to be a $\gamma$ Lipschitz continuous mapping if there exists a constant $\gamma>0$ such that

$$
\|T x-T y\| \leq \gamma\|x-y\|, \quad \forall x, y \in E .
$$

We denote by $F(T)$ the set of fixed points of $T$, that is, $F(T)=\{x \in E: T x=x\}$. For any nonempty set $\Xi \subset E \times E$, the symbol $\Xi \cap F(T) \neq \varnothing$ means that there exist $x^{*}, y^{*} \in$ $E$ such that $\left(x^{*}, y^{*}\right) \in \Xi$ and $\left\{x^{*}, y^{*}\right\} \subset F(T)$.

Remark 2.2. (1) If $\gamma=1$, then a $\gamma$-Lipschitz continuous mapping reduces to a nonexpansive mapping.

(2) If $\gamma \in(0,1)$, then a $\gamma$-Lipschitz continuous mapping reduces to a contractive mapping.

Definition 2.3. Let $A: E \times E \rightarrow E$ be a mapping. $A$ is said to be

(i) $\tau$-Lipschitz continuous in the first variable if there exists a constant $\tau>0$ such that, for $x, \tilde{x} \in E$,

$$
\|A(x, y)-A(\tilde{x}, \tilde{y})\| \leq \tau\|x-\tilde{x}\|, \quad \forall y, \tilde{y} \in E .
$$

(ii) $\alpha$-strongly accretive if there exists a constant $\alpha>0$ such that

$$
\left\langle A(x, y)-A(\tilde{x}, \tilde{y}), J_{q}(x-\tilde{x})\right\rangle \geq \alpha\|x-\tilde{x}\|^{q}, \quad \forall(x, y),(\tilde{x}, \tilde{y}) \in E \times E,
$$

or equivalently,

$$
\langle A(x, y)-A(\tilde{x}, \tilde{y}), J(x-\tilde{x})\rangle \geq \alpha\|x-\tilde{x}\|, \quad \forall(x, y),(\tilde{x}, \tilde{y}) \in E \times E .
$$

(iii) $\alpha$-inverse strongly accretive or $\alpha$-cocoercive if there exists a constant $\alpha>0$ such that

$$
\left\langle A(x, y)-A(\tilde{x}, \tilde{y}), J_{q}(x-\tilde{x})\right\rangle \geq \alpha\|A(x, y)-A(\tilde{x}, \tilde{y})\|^{q}, \quad \forall(x, y),(\tilde{x}, \tilde{y}) \in E \times E,
$$

or equivalently,

$$
\langle A(x, y)-A(\tilde{x}, \tilde{y}), J(x-\tilde{x})\rangle \geq \alpha\|A(x, y)-A(\tilde{x}, \tilde{y})\|, \quad \forall(x, y),(\tilde{x}, \tilde{y}) \in E \times E .
$$


(iv) $(\mu, v)$-relaxed cocoercive if there exist two constants $\mu \leq 0$ and $v>0$ such that

$$
\left\langle A(x, y)-A(\tilde{x}, \tilde{y}), J_{q}(x-\tilde{x})\right\rangle \geq(-\mu)\|A(x, y)-A(\tilde{x}, \tilde{y})\|^{q}+\nu\|x-\tilde{x}\|^{q}, \quad \forall(x, y),(\tilde{x}, \tilde{y}) \in E \times E .
$$

Remark 2.3. (1) Every $\alpha$-strongly accretive mapping is a $(\mu, \alpha)$-relaxed cocoercive for any positive constant $\mu$. But the converse is not true in general.

(2) The conception of the cocoercivity is applied in several directions, especially for solving variational inequality problems by using the auxiliary problem principle and projection methods [24]. Several classes of relaxed cocoercive variational inequalities have been investigated in $[18,23,25,26]$.

Definition 2.4. Let the set-valued mapping $M: \operatorname{dom}(M) \subset E \rightarrow 2^{E}$ be $m$-accretive. For any positive number $\rho>0$, the mapping $R_{(\rho, M)}: E \rightarrow \operatorname{dom}(M)$ defined by

$$
R_{(\rho, M)}(x)=(I+\rho M)^{-1}(x), \quad x \in E,
$$

is called the resolvent operator associated with $M$ and $\rho$, where $I$ is the identity operator on $E$.

Remark 2.4. Let $C \subset E$ be a nonempty closed convex set. If $E$ is a Hilbert space, and $M=\partial \varphi$, the subdifferential of the indicator function $\varphi$, that is,

$$
\phi(x)=\delta_{C}(x)= \begin{cases}0 & \text { if } x \in C, \\ +\infty & \text { otherwise }\end{cases}
$$

then $R_{(\rho, M)}=P_{C}$, the metric projection operator from $E$ onto $C$.

In order to estimate of convergence rates for sequence, we need the following definition.

Definition 2.5. Let a sequence $\left\{x_{n}\right\}$ converge strongly to $x^{*}$. The sequence $\left\{x_{n}\right\}$ is said to be at least linear convergence if there exists a constant $\varrho \in(0,1)$ such that

$$
\left\|x_{n+1}-x^{*}\right\| \leq \varrho\left\|x_{n}-x^{*}\right\| .
$$

Lemma 2.1.[27] Let the set-valued mapping $M: \operatorname{dom}(M) \subset E \rightarrow 2^{E}$ be $m$-accretive. Then the resolvent operator $R_{(\rho, M)}$ is single valued and nonexpansive for all $\rho>0$ :

Lemma 2.2.[28] Let $\left\{a_{n}\right\}$ and $\left\{b_{n}\right\}$ be two nonnegative real sequences satisfying the following conditions:

$$
a_{n+1} \leq\left(1-\lambda_{n}\right) a_{n}+b_{n}, \quad \forall n \geq n_{0},
$$

for some $n_{0} \in N,\left\{\lambda_{n}\right\} \subset(0,1)$ with $\sum_{n=0}^{\infty} \lambda_{n}=\infty$ and $b_{n}=0\left(\lambda_{n}\right)$. Then $\lim _{n \rightarrow \infty} a_{n}=$ 0 .

Lemma 2.3.[29] Let $E$ be a real $q$-uniformly Banach space. Then there exists a constant $c_{q}>0$ such that

$$
\|x+y\|^{q} \leq\|x\|^{q}+q\left\langle y, J_{q}(x)\right\rangle+c_{q}\|y\|^{q}, \quad \forall x, y \in E .
$$

\section{Existence and uniqueness of solutions for (SGQVI)}

In this section, we shall investigate the existence and uniqueness of solutions for (SGQVI) in $q$-uniformly smooth Banach space under some suitable conditions.

Theorem 3.1. Let $\rho_{1}, \rho_{2}$ be two positive constants, and $\left(x^{*}, y^{*}\right) \in E \times E$. Then $\left(x^{*}, y^{*}\right)$ is a solution of the problem (2.1) if and only if 


$$
\left\{\begin{array}{l}
x^{*}=R_{\left(\rho_{1}, M_{1}\right)}\left(y^{*}-\rho_{1} A_{1}\left(y^{*}, x^{*}\right)\right) \\
\gamma^{*}=R_{\left(\rho_{2}, M_{2}\right)}\left(x^{*}-\rho_{2} A_{2}\left(x^{*}, y^{*}\right)\right)
\end{array}\right.
$$

Proof. It directly follows from Definition 2.4. This completes the proof. $\square$

Theorem 3.2. Let $E$ be a real $q$-uniformly smooth Banach space. Let $M_{2}: E \rightarrow 2^{E}$ be $m$-accretive mapping, $A_{2}: E \times E \rightarrow E$ be $\left(\mu_{2}, v_{2}\right)$-relaxed cocoercive and Lipschitz continuous in the first variable with constant $\tau_{2}$. Then, for each $x \in E$, the mapping $R_{\left(\rho_{2}, \mathrm{M}_{2}\right)}\left(x-\rho_{2} A_{2}(x, \cdot)\right): E \rightarrow E$ has at most one fixed point. If

$$
1-q \rho_{2} \nu_{2}+q \rho_{2} \mu_{2} \tau_{2}^{q}+c_{q} \rho_{2}^{q} \tau_{2}^{q} \geq 0
$$

then the implicit function $y(x)$ determined by

$$
y(x)=R_{\left(\rho_{2}, M_{2}\right)}\left(x-\rho_{2} A_{2}(x, y(x))\right),
$$

is continuous on $E$.

Proof. Firstly, we show that, for each $x \in E$, the mapping $R_{\left(\rho_{2}, \mathrm{M}_{2}\right)}\left(x-\rho_{2} A_{2}(x, \cdot)\right): E \rightarrow E$ has at most one fixed point. Assume that $y, \tilde{y} \in E$ such that

$$
\begin{aligned}
& y=R_{\left(\rho_{2}, M_{2}\right)}\left(x-\rho_{2} A_{2}(x, y)\right), \\
& \tilde{y}=R_{\left(\rho_{2}, M_{2}\right)}\left(x-\rho_{2} A_{2}(x, \tilde{y})\right) .
\end{aligned}
$$

Since $A_{2}$ is Lipschitz continuous in the first variable with constant $\tau_{2}$, then

$$
\begin{aligned}
\|y-\tilde{y}\| & =\left\|R_{\left(\rho_{2}, M_{2}\right)}\left(x-\rho_{2} A_{2}(x, y)\right)-R_{\left(\rho_{2}, M_{2}\right)} 2,\left(x-\rho_{2} A_{2}(x, \tilde{y})\right)\right\| \\
& \leq\left\|x-\rho_{2} A_{2}(x, y)-\left(x-\rho_{2} A_{2}(x, \tilde{y})\right)\right\| \\
& \left.=\rho_{2} \| A_{2}(x, y)-A_{2}(x, \tilde{y})\right) \| \\
& \leq \rho_{2} \tau_{2}\|x-x\|=0 .
\end{aligned}
$$

Therefore, $y=\tilde{y}$.

On the other hand, for any sequence $\left\{x_{n}\right\} \subset E, x_{0} \in E, x_{n} \rightarrow x_{0}$ as $n \rightarrow \infty$ : Since $A_{2}$ : $E \times E \rightarrow E$ is $\left(\mu_{2}, v_{2}\right)$-relaxed cocoercive and Lipschitz continuous in the first variable with constant $\tau_{2}$, one has

$$
\begin{aligned}
L & =\left\|A_{2}\left(x_{n}, \gamma\left(x_{n}\right)\right)-A_{2}\left(x_{0}, \gamma\left(x_{0}\right)\right)\right\|^{q} \\
& \leq \tau_{2}^{q}\left\|x_{n}-x_{0}\right\|^{q}, \\
Q & =\left\langle A_{2}\left(x_{n}, \gamma\left(x_{n}\right)\right)-A_{2}\left(x_{0}, \gamma\left(x_{0}\right)\right), J_{q}\left(x_{n}-x_{0}\right)\right\rangle \\
& \geq\left(-\mu_{2}\right)\left\|A_{2}\left(x_{n}, \gamma\left(x_{n}\right)\right)-A_{2}\left(x_{0}, \gamma\left(x_{0}\right)\right)\right\|^{q}+v_{2}\left\|x_{n}-x_{0}\right\|^{q} \\
& \geq\left(-\mu_{2} \tau_{2}^{q}+v_{2}\right)\left\|x_{n}-x_{0}\right\|^{q} .
\end{aligned}
$$

As a consequence, we have, by Lemma 2.1,

$$
\begin{aligned}
\left\|y\left(x_{n}\right)-y\left(x_{0}\right)\right\| & =\left\|R_{\left(\rho_{2}, M_{2}\right)}\left(x_{n}-\rho_{2} A_{2}\left(x_{n}, \gamma\left(x_{n}\right)\right)\right)-R_{\left(\rho_{2}, M_{2}\right)}\left(x_{0}-\rho_{2} A_{2}\left(x_{0}, \gamma\left(x_{0}\right)\right)\right)\right\| \\
& \leq\left\|x_{n}-\rho_{2} A_{2}\left(x_{n}, \gamma\left(x_{n}\right)\right)-\left(x_{0}-\rho_{2} A_{2}\left(x_{0}, \gamma\left(x_{0}\right)\right)\right)\right\| \\
& =\left\|\left(x_{n}-x_{0}\right)-\rho_{2}\left(A_{2}\left(x_{n}, \gamma\left(x_{n}\right)\right)-A_{2}\left(x_{0}, \gamma\left(x_{0}\right)\right)\right)\right\| \\
& \leq \sqrt[q]{\left\|x_{n}-x_{0}\right\|^{q}-q \rho_{2} Q+c_{q} \rho_{2}^{q} L} \\
& \leq \sqrt[q]{\left\|x_{n}-x_{0}\right\|^{q}-q \rho_{2}\left(-\mu_{2} \tau_{2}^{q}+v_{2}\right)\left\|x_{n}-x_{0}\right\|^{q}+c_{q} \rho_{2}^{q} \tau_{2}^{q}\left\|x_{n}-x_{0}\right\|^{q}} \\
& =\sqrt[q]{1-q \rho_{2} v_{2}+q \rho_{2} \mu_{2} \tau_{2}^{q}+c_{q} \rho_{2}^{q} \tau_{2}^{q}}\left\|x_{n}-x_{0}\right\| .
\end{aligned}
$$


Together with (3.2), it yields that the implicit function $y(x)$ is continuous on $E$. This completes the proof. $\square$

Theorem 3.3. Let $E$ be a real $q$-uniformly smooth Banach space. Let $M_{2}: E \rightarrow 2^{E}$ be $m$-accretive mapping, $A_{2}: E \times E \rightarrow E$ be $\alpha_{2}$-strong accretive and Lipschitz continuous in the first variable with constant $\tau_{2}$. Then, for each $x \in E$, the mapping $R_{\left(\rho_{2}, M_{2}\right)}\left(x-\rho_{2} A_{2}(x, \cdot)\right): E \rightarrow E$ has at most one fixed point. If $1-q \rho_{2} \alpha_{2}+c_{q} \rho_{2}^{q} \tau_{2}^{q} \geq 0$, then the implicit function $y(x)$ determined by

$$
y(x)=R_{\left(\rho_{2}, M_{2}\right)}\left(x-\rho_{2} A_{2}(x, y(x))\right),
$$

is continuous on $E$.

Proof. The proof is similar to Theorem 3.2 and so the proof is omitted. This completes the proof.

Theorem 3.4. Let $E$ be a real $q$-uniformly smooth Banach space. Let $M_{i}: E \rightarrow 2^{E}$ be $m$ - accretive mapping, $A_{i}: E \times E \rightarrow E$ be $\left(\mu_{i}, v_{i}\right)$-relaxed cocoercive and Lipschitz continuous in the first variable with constant $\tau_{i}$ for $i \in\{1,2\}$. If $1-q \rho_{2} v_{2}+q \rho_{2} \mu_{2} \tau_{2}^{q}+c_{q} \rho_{2}^{q} \tau_{2}^{q} \geq 0$, and

$$
0 \leq \prod_{i=1}^{2}\left(1-q \rho_{i} \nu_{i}+q \rho_{i} \mu_{i} \tau_{i}^{q}+c_{q} \rho_{i}^{q} \tau_{i}^{q}\right)<1 .
$$

Then the solutions set $\Omega$ of (SGQVI) is nonempty. Moreover, $\Omega$ is a singleton.

Proof. By Theorem 3.2, we define a mapping $P: E \rightarrow E$ by

$$
\begin{gathered}
P(x)=R_{\left(\rho_{1}, M_{1}\right)}\left(y(x)-\rho_{1} A_{1}(y(x), x)\right), \\
\gamma(x)=R_{\left(\rho_{2}, M_{2}\right)}\left(x-\rho_{2} A_{2}(x, y(x))\right), \quad \forall x \in E .
\end{gathered}
$$

Since $A_{i}: E \times E \rightarrow E$ are $\left(\mu_{i}, v_{i}\right)$-relaxed cocoercive and Lipschitz continuous in the first variable with constant $\tau_{i}$ for $i \in\{1,2\}$, one has, for any $x, \tilde{x} \in E$,

$$
\begin{aligned}
L_{1} & =\left\|A_{1}(y(x), x)-A_{1}(y(\tilde{x}), \tilde{x})\right\|^{q} \\
& \leq \tau_{1}^{q}\|y(x)-\gamma(\tilde{x})\|^{q}, \\
Q_{1} & =\left\langle A_{1}(\gamma(x), x)-A_{1}(y(\tilde{x}), \tilde{x}), J_{q}(y(x)-\gamma(\tilde{x}))\right\rangle \\
& \geq\left(-\mu_{1}\right)\left\|A_{1}(\gamma(x), x)-A_{1}(\gamma(\tilde{x}), \tilde{x})\right\|^{q}+\nu_{1}\|y(x)-\gamma(\tilde{x})\|^{q} \\
& \geq\left(-\mu_{1} \tau_{1}^{q}+v_{1}\right)\|y(x)-\gamma(\tilde{x})\|^{q}, \\
L_{2} & =\left\|A_{2}(x, \gamma(x))-A_{2}(\tilde{x}, \gamma(\tilde{x}))\right\|^{q} \\
& \leq \tau_{2}^{q}\|x-\tilde{x}\|^{q},
\end{aligned}
$$

and

$$
\begin{aligned}
Q_{2} & =\left\langle A_{2}(x, y(x))-A_{2}(\tilde{x}, y(\tilde{x})), J_{q}(x-\tilde{x})\right\rangle \\
& \geq\left(-\mu_{2}\right)\left\|A_{2}(x, y(x))-A_{2}(\tilde{x}, y(\tilde{x}))\right\|^{q}+\nu_{2}\|x-\tilde{x}\|^{q} \\
& \geq\left(-\mu_{2} \tau_{2}^{q}+v_{2}\right)\|x-\tilde{x}\|^{q} .
\end{aligned}
$$

From both Lemma 2.1 and Theorem 3.1, we get

$$
\begin{aligned}
\|P(x)-P(\tilde{x})\| & =\left\|R_{\left(\rho_{1}, M_{1}\right)}\left(\gamma(x)-\rho_{1} A_{1}(\gamma(x), x)\right)-R_{\left(\rho_{1}, M_{1}\right)}\left(y(\tilde{x})-\rho_{1} A_{1}(\gamma(\tilde{x}), \tilde{x})\right)\right\| \\
& \leq\left\|\left(y(x)-\rho_{1} A_{1}(y(x), x)\right)-\left(y(\tilde{x})-\rho_{1} A_{1}(y(\tilde{x}), \tilde{x})\right)\right\| \\
& \left.\left.=\|(y(x)-\gamma(\tilde{x}))-\rho_{1}\left(A_{1}(y(x), x)\right)-A_{1}(\gamma(\tilde{x}), \tilde{x})\right)\right) \| \\
& \leq \sqrt[q]{\|y(x)-\gamma(\tilde{x})\| q-q \rho_{1} Q_{1}+c_{q} \rho_{1}^{q} L_{1}} \\
& \leq \sqrt[q]{1-q \rho_{1}\left(-\mu_{1} \tau_{1}^{q}+v_{1}\right)+c_{q} \rho_{1}^{q} \tau_{1}^{q}}\|y(x)-\gamma(\tilde{x})\| .
\end{aligned}
$$


Note that

$$
\begin{aligned}
\|y(x)-y(\tilde{x})\| & =\left\|R_{\left(\rho_{2}, \mathrm{M}_{2}\right)}\left(x-\rho_{2} A_{2}(x, y(x))\right)-R_{\left(\rho_{2}, \mathrm{M}_{2}\right)}\left(\tilde{x}-\rho_{2} A_{2}(\tilde{x}, y(\tilde{x}))\right)\right\| \\
& \leq\left\|\left(x-\rho_{2} A_{2}(x, y(x))\right)-\left(\tilde{x}-\rho_{2} A_{2}(\tilde{x}, y(\tilde{x}))\right)\right\| \\
& \left.=\|(x-\tilde{x})-\rho_{2}\left(A_{2}(x, y(x))\right)-A_{2}(\tilde{x}, y(\tilde{x}))\right) \| \\
& \leq \sqrt[q]{\|x-\tilde{x}\|^{q}-q \rho_{2} Q_{2}+c_{q} \rho_{2}^{q} L_{2}} \\
& \leq \sqrt[q]{1-q \rho_{2}\left(-\mu_{2} \tau_{2}^{q}+v_{2}\right)+c_{q} \rho_{2}^{q} \tau_{2}^{q}}\|x-\tilde{x}\| .
\end{aligned}
$$

Therefore, we obtain

$$
\begin{aligned}
\|P(x)-P(\tilde{x})\| & \leq \prod_{i=1}^{2} \sqrt[q]{1-q \rho_{i}\left(-\mu_{i} \tau_{i}^{q}+v_{i}\right)+c_{q} \rho_{i}^{q} \tau_{i}^{q}}|| x-\tilde{x} \| \\
& =\prod_{i=1}^{2} \sqrt[q]{1-q \rho_{i} v_{i}+q \rho_{i} \mu_{i} \tau_{i}^{q}+c_{q} \rho_{i}^{q} \tau_{i}^{q}}\|x-\tilde{x}\| .
\end{aligned}
$$

From (3.3), this yields that the mapping $P$ is contractive. By Banach contraction principle, there exists a unique $x^{*} \in E$ such that $P\left(x^{*}\right)=x^{*}$. Therefore, from Theorem 3.2, there exists an unique $\left(x^{*}, y^{*}\right) \in \Omega$, where $y^{*}=y\left(x^{*}\right)$. This completes the proof. $\square$

Theorem 3.5. Let $E$ be a real $q$-uniformly smooth Banach space. Let $M_{i}: E \rightarrow 2^{E}$ be $m$ - accretive mapping, $A_{i}: E \times E \rightarrow E$ be $\alpha_{i}$-strong accretive and Lipschitz continuous in the first variable with constant $\tau_{i}$ for $i \in\{1,2\}$. If $1-q \rho_{2} \alpha_{2}+c_{q} \rho_{2}^{q} \tau_{2}^{q} \geq 0$, and

$$
0 \leq \prod_{i=1}^{2}\left(1-q \rho_{i} \alpha_{i}+c_{q} \rho_{i}^{q} \tau_{i}^{q}\right)<1
$$

Then the solutions set $\Omega$ of (SGQVI) is nonempty. Moreover, $\Omega$ is a singleton.

Proof. It is easy to know that Theorem 3.5 follows from Remark 2.3 and Theorem 3.4 and so the proof is omitted. This completes the proof. $\square$

In order to show the existence of $\rho_{i} i=1,2$, we give the following examples.

Example 3.1. Let $E$ be a 2-uniformly smooth space, and let $M_{1}, M_{2}, A_{1}$ and $A_{2}$ be the same as Theorem 3.4. Then there exist $\rho_{1}, \rho_{2}>0$ such that (3.3), where

$$
\rho_{i} \in\left(0, \frac{2 \nu_{i}-2 \mu_{i} \tau_{i}^{2}}{c_{2} \tau_{i}^{2}}\right), \quad v_{i}>\mu_{i} \tau_{i}^{2}, \quad\left(\mu_{i} \tau_{i}^{2}-v_{i}\right)^{2}<c_{2} \tau_{i}^{2}, \quad i=1,2
$$

or

$$
\begin{gathered}
\rho_{i} \in\left(0, \frac{v_{i}-\mu_{i} \tau_{i}^{2}-\sqrt{\left(v_{i}-\mu_{i} \tau_{i}^{2}\right)^{2}-c_{2} \tau_{i}^{2}}}{c_{2} \tau_{i}^{2}}\right) \cup\left(\frac{v_{i}-\mu_{i} \tau_{i}^{2}+\sqrt{\left(v_{i}-\mu_{i} \tau_{i}^{2}\right)^{2}-c_{2} \tau_{i}^{2}}}{c_{2} \tau_{i}^{2}}, \frac{2 v_{i}-2 \mu_{i} \tau_{i}^{2}}{c_{2} \tau_{i}^{2}}\right), \\
v_{i}>\mu_{i} \tau_{i}^{2}, \quad\left(\mu_{i} \tau_{i}^{2}-v_{i}\right)^{2} \geq c_{2} \tau_{i}^{2}, \quad i=1,2 .
\end{gathered}
$$

Example 3.2. Let $E$ be a 2-uniformly smooth space, and let $M_{1}, M_{2}, A_{1}$ and $A_{2}$ be the same as Theorem 3.5. Then there exist $\rho_{1}, \rho_{2}>0$ such that (3.4), where

$$
\rho_{i} \in\left(0, \frac{2 \alpha_{i}}{c_{2} \tau_{i}^{2}}\right), \quad \alpha_{i}<\tau_{i} \sqrt{c_{2}}, \quad i=1,2,
$$


or

$$
\rho_{i} \in\left(0, \frac{\alpha_{i}-\sqrt{\alpha_{i}^{2}-c_{2} \tau_{i}^{2}}}{c_{2} \tau_{i}^{2}}\right) \cup\left(\frac{\alpha_{i}-\sqrt{\alpha_{i}^{2}+c_{2} \tau_{i}^{2}}}{c_{2} \tau_{i}^{2}}, \frac{2 \alpha_{i}}{c_{2} \tau_{i}^{2}}\right), \quad \alpha_{i} \geq \tau_{i} \sqrt{c_{2}}, \quad i=1,2
$$

\section{Algorithms and convergence analysis}

In this section, we introduce two-step iterative sequences for the problem (SGQVI) and a non-linear mapping, and then explore the convergence analysis of the iterative sequences generated by the algorithms.

Let $T: E \rightarrow E$ be a nonlinear mapping and the fixed points set $F(T)$ of $T$ be a nonempty set. In order to introduce the iterative algorithm, we also need the following lemma.

Lemma 4.1. Let $E$ be a real $q$-uniformly smooth Banach space, $\rho_{1}, \rho_{2}$ be two positive constants. If $\left(x^{*}, y^{*}\right) \in \Omega$ and $\left\{x^{*}, y^{*}\right\} \subset F(T)$, then

$$
\left\{\begin{array}{l}
x^{*}=T R_{\left(\rho_{1}, M_{1}\right)}\left(y^{*}-\rho_{1} A_{1}\left(y^{*}, x^{*}\right)\right), \\
y^{*}=T R_{\left(\rho_{2}, M_{2}\right)}\left(x^{*}-\rho_{2} A_{2}\left(x^{*}, y^{*}\right)\right) .
\end{array}\right.
$$

Proof. It directly follows from Theorem 3.1. This completes the proof. $\square$

Now we introduce the following iterative algorithms for finding a common element of the set of solutions to a (SGQVI) problem (2.1) and the set of fixed points of a Lipschtiz mapping.

Algorithm 4.1. Let $E$ be a real $q$-uniformly smooth Banach space, $\rho_{1}, \rho_{2}>0$, and let $T: E \rightarrow E$ be a nonlinear mapping. For any given points $x_{0}, y_{0} \in E$, define sequences $\left\{x_{n}\right\}$ and $\left\{y_{n}\right\}$ in $E$ by the following algorithm:

$$
\left\{\begin{array}{l}
y_{n}=\left(1-\beta_{n}\right) x_{n}+\beta_{n} T R_{\left(\rho_{2} M_{2}\right)},\left(x_{n}-\rho_{2} A_{2}\left(x_{n}, y_{n}\right)\right), \\
x_{n+1}=\left(1-\alpha_{n}\right) x_{n}+\alpha_{n} T R_{\left(\rho_{1} M_{1}\right)}\left(y_{n}-\rho_{1} A_{1}\left(y_{n}, x_{n}\right)\right), \quad n=0,1,2, \ldots,
\end{array}\right.
$$

where $\left\{\alpha_{n}\right\}$ and $\left\{\beta_{n}\right\}$ are sequences in $[0,1]$.

Algorithm 4.2. Let $E$ be a real $q$-uniformly smooth Banach space, $\rho_{1}, \rho_{2}>0$, and let $T: E \rightarrow E$ be a nonlinear mapping. For any given points $x_{0}, y_{0} \in E$, define sequences $\left\{x_{n}\right\}$ and $\left\{y_{n}\right\}$ in $E$ by the following algorithm:

$$
\left\{\begin{array}{l}
y_{n}=\operatorname{TR}_{\left(\rho_{2}, M_{2}\right)}\left(x_{n}-\rho_{2} A_{2}\left(x_{n}, y_{n}\right)\right), \\
x_{n+1}=\left(1-\alpha_{n}\right) x_{n}+\alpha_{n} T R_{\left(\rho_{1}, M_{1}\right)}\left(y_{n}-\rho_{1} A_{1}\left(y_{n}, x_{n}\right)\right), \quad n=0,1,2, \ldots,
\end{array}\right.
$$

where $\left\{\alpha_{n}\right\}$ is a sequence in $[0,1]$.

Remark 4.1. If $A_{1}=A_{2}=A, E=H$ is a Hilbert space, and $M_{1}(x)=M_{2}(x)=\partial \varphi(x)$ for all $x \in E$, where $\varphi: E \rightarrow R \cup\{+\infty\}$ is a proper, convex and lower semicontinuous functional, and $\partial \varphi$ denotes the subdifferential operator of $\varphi$, then Algorithm 4.1 is reduced to the Algorithm (I) of [18].

Theorem 4.1. Let $E$ be a real $q$-uniformly smooth Banach space, and $A_{1}, A_{2}, M_{1}$ and $M_{2}$ be the same as in Theorem 3.4, and let $T$ be a $\kappa$-Lipschitz continuous mapping. Assume that $\Omega \cap F(T) \neq \varnothing,\left\{\alpha_{n}\right\}$ and $\left\{\beta_{n}\right\}$ are sequences in $[0,1]$ and satisfy the following conditions:

(i) $\sum_{i=0}^{\infty} \alpha_{n}=\infty$; 
(ii) $\lim _{n \rightarrow \infty} \beta_{n}=1$;

(iii) $0<\kappa \sqrt[q]{1-q \rho_{i} \nu_{i}+q \rho_{i} \mu_{i} \tau_{i}^{q}+c_{q} \rho_{i}^{q} \tau_{i}^{q}}<1, i=1,2$.

Then the sequences $\left\{x_{n}\right\}$ and $\left\{y_{n}\right\}$ generated by Algorithm 4.1 converge strongly to $x^{*}$ and $y^{*}$, respectively, such that $\left(x^{*}, y^{*}\right) \in$ and $\left\{x^{*}, y^{*}\right\} \subset F(T)$.

Proof. Let $\left(x^{*}, y^{*}\right) \in \Omega$ and $\left\{x^{*}, y^{*}\right\} \subset F(T)$. Then, from (4.1), one has

$$
\left\{\begin{array}{l}
x^{*}=T R_{\left(\rho_{1}, M_{1}\right)}\left(y^{*}-\rho_{1} A_{1}\left(y^{*}, x^{*}\right)\right), \\
y^{*}=\operatorname{TR}_{\left(\rho_{2}, M_{2}\right)}\left(x^{*}-\rho_{2} A_{2}\left(x^{*}, y^{*}\right)\right) .
\end{array}\right.
$$

Since $T$ is a $\kappa$-Lipschitz continuous mapping, and from both (4.2) and (4.3), we have

$$
\begin{aligned}
\left\|x_{n+1}-x^{*}\right\|= & \left\|\alpha_{n}\left(T R_{\left(\rho_{1}, M_{1}\right)}\left(y_{n}-\rho_{1} A_{1}\left(y_{n}, x_{n}\right)\right)-x^{*}\right)+\left(1-\alpha_{n}\right)\left(x_{n}-x^{*}\right)\right\| \\
= & \| \alpha_{n}\left(T R_{\left(\rho_{1}, M_{1}\right)}\left(y_{n}-\rho_{1} A_{1}\left(y_{n}, x_{n}\right)\right)-T R_{\left(\rho_{1}, M_{1}\right)}\left(y^{*}-\rho_{1} A_{1}\left(y^{*}, x^{*}\right)\right)\right) \\
& +\left(1-\alpha_{n}\right)\left(x_{n}-x^{*}\right) \| \\
\leq & \alpha_{n}\left\|T R_{\left(\rho_{1}, M_{1}\right)}\left(y_{n}-\rho_{1} A_{1}\left(y_{n}, x_{n}\right)\right)-T R_{\left(\rho_{1}, M_{1}\right)}\left(\gamma^{*}-\rho_{1} A_{1}\left(y^{*}, x^{*}\right)\right)\right\| \\
& +\left(1-\alpha_{n}\right)\left\|x_{n}-x^{*}\right\| \\
\leq & \alpha_{n} \kappa\left\|R_{\left(\rho_{1}, M_{1}\right)}\left(y_{n}-\rho_{1} A_{1}\left(y_{n}, x_{n}\right)\right)-R_{\left(\rho_{1}, M_{1}\right)}\left(y^{*}-\rho_{1} A_{1}\left(\gamma^{*}, x^{*}\right)\right)\right\| \\
& +\left(1-\alpha_{n}\right)\left\|x_{n}-x^{*}\right\| \\
\leq & \alpha_{n} \kappa\left\|\left(y_{n}-\gamma^{*}\right)-\rho_{1}\left(A_{1}\left(y_{n}, x_{n}\right)-A_{1}\left(\gamma^{*}, x^{*}\right)\right)\right\|+\left(1-\alpha_{n}\right)\left\|x_{n}-x^{*}\right\| .
\end{aligned}
$$

For each $i \in\{1,2\}, A_{i}: E \times E \rightarrow E$ are $\left(\mu_{i}, v_{i}\right)$-relaxed cocoercive and Lipschitz continuous in the first variable with constant $\tau_{i}$, then

$$
\begin{aligned}
\tilde{L}_{1} & =\left\|A_{1}\left(y_{n}, x_{n}\right)-A_{1}\left(y^{*}, x^{*}\right)\right\|^{q} \\
& \leq \tau_{1}^{q}\left\|y_{n}-\gamma^{*}\right\|^{q}, \\
\tilde{Q}_{1} & =\left\langle A_{1}\left(y_{n}, x_{n}\right)-A_{1}\left(\gamma^{*}, x^{*}\right), J_{q}\left(y_{n}-y^{*}\right)\right\rangle \\
& \geq\left(-\mu_{1}\right)\left\|A_{1}\left(y_{n}, x_{n}\right)-A_{1}\left(\gamma^{*}, x^{*}\right)\right\|^{q}+v_{1}\left\|y_{n}-y^{*}\right\|^{q} \\
& \geq-\mu_{1} \tau_{1}^{q}\left\|y_{n}-\gamma^{*}\right\|^{q}+v_{1}\left\|y_{n}-\gamma^{*}\right\|^{q} \\
& =\left(-\mu_{1} \tau_{1}^{q}+v_{1}\right)\left\|y_{n}-\gamma^{*}\right\|^{q}, \\
\tilde{L}_{2} & =\left\|A_{2}\left(x_{n}, y_{n}\right)-A_{2}\left(x^{*}, y^{*}\right)\right\|^{q} \\
& \leq \tau_{2}^{q}\left\|x_{n}-x^{*}\right\|^{q},
\end{aligned}
$$

and so

$$
\begin{aligned}
\tilde{Q}_{2} & =\left\langle A_{2}\left(x_{n}, y_{n}\right)-A_{2}\left(x^{*}, y^{*}\right), J_{q}\left(x_{n}-x^{*}\right)\right\rangle \\
& \geq\left(-\mu_{2}\right)\left\|A_{2}\left(x_{n}, y_{n}\right)-A_{2}\left(x^{*}, y^{*}\right)\right\|^{q}+v_{2}\left\|x_{n}-x^{*}\right\|^{q} \\
& \geq-\mu_{2} \tau_{2}^{q}\left\|x_{n}-x^{*}\right\|^{q}+v_{2}\left\|x_{n}-x^{*}\right\|^{q} \\
& =\left(-\mu_{2} \tau_{2}^{q}+v_{2}\right)\left\|x_{n}-x^{*}\right\|^{q} .
\end{aligned}
$$

Furthermore, by Lemma 2.1, one can obtain

$$
\begin{aligned}
\left\|\left(y_{n}-\gamma^{*}\right)-\rho_{1}\left(A_{1}\left(y_{n}, x_{n}\right)-A_{1}\left(\gamma^{*}, x^{*}\right)\right)\right\| & =\sqrt[q]{\left\|y_{n}-\gamma^{*} \mid\right\|^{q}-q \rho_{1} \tilde{Q}_{1}+c_{q} \rho_{1}^{q} \tilde{L}_{1}} \\
& \leq \sqrt[q]{1-q \rho_{1}\left(-\mu_{1} \tau_{1}^{q}+v_{1}\right)+c_{q} \rho_{1}^{q} \tau_{1}^{q}}\left\|y_{n}-\gamma^{*}\right\| \\
& =\sqrt[q]{1-q \rho_{1} v_{1}+q \rho_{1} \mu_{1} \tau_{1}^{q}+c_{q} \rho_{1}^{q} \tau_{1}^{q}}\left\|y_{n}-\gamma^{*}\right\|
\end{aligned}
$$


and consequently,

$$
\begin{aligned}
\left\|\left(x_{n}-x^{*}\right)-\rho_{2}\left(A_{2}\left(x_{n}, y_{n}\right)-A_{2}\left(x^{*}, y^{*}\right)\right)\right\| & =\sqrt[q]{\left\|x_{n}-x^{*}\right\| \|^{q}-q \rho_{2} \tilde{Q}_{2}+c_{q} \rho_{2}^{q} \tilde{L}_{2}} \\
& \leq \sqrt[q]{1-q \rho_{2} v_{2}+q \rho_{2} \mu_{2} \tau_{2}^{q}+c_{q} \rho_{2}^{q} \tau_{2}^{q}}\left\|x_{n}-x^{*}\right\| .
\end{aligned}
$$

Note that

$$
\begin{aligned}
\left\|y_{n}-\gamma^{*}\right\|= & \left\|\left(1-\beta_{n}\right)\left(x_{n}-\gamma^{*}\right)+\beta_{n}\left(T R_{\left(\rho_{2}, M_{2}\right)}\left(x_{n}-\rho_{2} A_{2}\left(x_{n}, y_{n}\right)\right)-T y^{*}\right)\right\| \\
\leq & \left(1-\beta_{n}\right)\left\|x_{n}-\gamma^{*}\right\|+\beta_{n}\left\|T R_{\left(\rho_{2}, M_{2}\right)}\left(x_{n}-\rho_{2} A_{2}\left(x_{n}, y_{n}\right)\right)-T \gamma^{*}\right\| \\
\leq & \left(1-\beta_{n}\right)\left\|x_{n}-\gamma^{*}\right\|+\beta_{n} \kappa\left\|R_{\left(\rho_{2}, M_{2}\right)}\left(x_{n}-\rho_{2} A_{2}\left(x_{n}, y_{n}\right)\right)-y^{*}\right\| \\
= & \beta_{n} \kappa\left\|R_{\left(\rho_{2}, M_{2}\right)}\left(x_{n}-\rho_{2} A_{2}\left(x_{n}, y_{n}\right)\right)-R_{\left(\rho_{2}, M_{2}\right)}\left(x^{*}-\rho_{2} A_{2}\left(x^{*}, y^{*}\right)\right)\right\| \\
& +\left(1-\beta_{n}\right)\left\|x_{n}-\gamma^{*}\right\| \\
\leq & \beta_{n} \kappa\left\|\left(x_{n}-x^{*}\right)-\rho_{2}\left(A_{2}\left(x_{n}, y_{n}\right)-A_{2}\left(x^{*}, \gamma^{*}\right)\right)\right\|+\left(1-\beta_{n}\right)\left\|x_{n}-\gamma^{*}\right\| \\
\leq & \beta_{n} \kappa \sqrt[q]{1-q \rho_{2} v_{2}+q \rho_{2} \mu_{2} \tau_{2}^{q}+c_{q} \rho_{2}^{q} \tau_{2}^{q}}\left\|x_{n}-x^{*}\right\|+\left(1-\beta_{n}\right)\left\|x_{n}-\gamma^{*}\right\| \\
\leq & \left(\beta_{n} \kappa \sqrt[q]{1-q \rho_{2} v_{2}+q \rho_{2} \mu_{2} \tau_{2}^{q}+c_{q} \rho_{2}^{q} \tau_{2}^{q}}+1-\beta_{n}\right)\left\|x_{n}-x^{*}\right\|+\left(1-\beta_{n}\right)\left\|x^{*}-\gamma^{*}\right\| .
\end{aligned}
$$

Therefore, we have

$$
\begin{aligned}
\left\|x_{n+1}-x^{*}\right\| \leq & \alpha_{n} \kappa\left\|\left(y_{n}-\gamma^{*}\right)-\rho_{1}\left(A_{1}\left(y_{n}, x_{n}\right)-A_{1}\left(\gamma^{*}, x^{*}\right)\right)\right\|+\left(1-\alpha_{n}\right)\left\|x_{n}-x^{*}\right\| \\
\leq & \alpha_{n} \kappa \sqrt[q]{1-q \rho_{1} v_{1}+q \rho_{1} \mu_{1} \tau_{1}^{q}+c_{q} \rho_{1}^{q} \tau_{1}^{q}}\left\|y_{n}-\gamma^{*}\right\|+\left(1-\alpha_{n}\right)\left\|x_{n}-x^{*}\right\| \\
\leq & {\left[\alpha _ { n } \kappa \sqrt [ q ] { 1 - q \rho _ { 1 } v _ { 1 } + q \rho _ { 1 } \mu _ { 1 } \tau _ { 1 } ^ { q } + c _ { q } \rho _ { 1 } ^ { q } \tau _ { 1 } ^ { q } } \left(\beta_{n} \kappa \sqrt[q]{1-q \rho_{2} v_{2}+q \rho_{2} \mu_{2} \tau_{2}^{q}+c_{q} \rho_{2}^{q} \tau_{2}^{q}}+1-\right.\right.} \\
& \left.\left.\beta_{n}\right)+1-\alpha_{n}\right]\left\|x_{n}-x^{*}\right\|+\alpha_{n} \kappa\left(1-\beta_{n}\right) \sqrt[q]{1-q \rho_{1} v_{1}+q \rho_{1} \mu_{1} \tau_{1}^{q}+c_{q} \rho_{1}^{q} \tau_{1}^{q}}\left\|x^{*}-\gamma^{*}\right\| .
\end{aligned}
$$

Set $\iota=\max \left\{\sqrt[q]{1-q \rho_{i} v_{i}+q \rho_{i} \mu_{i} \tau_{i}^{q}+c_{q} \rho_{i}^{q} \tau_{i}^{q}}: i=1,2\right\}$. So the above inequality can be written as follows:

$$
\left\|x_{n+1}-x^{*}\right\| \leq\left\{1-\alpha_{n}\left[1-\kappa \iota\left(1-\beta_{n}(1-\kappa \iota)\right)\right]\right\}\left\|x_{n}-x^{*}\right\|+\alpha_{n} \kappa \iota\left(1-\beta_{n}\right)\left\|x^{*}-\gamma^{*}\right\| .
$$

Taking $a_{n}=\left\|x_{n}-x^{*}\right\|, \lambda_{n}=\alpha_{n}\left[1-\kappa \iota\left(1-\beta_{n}(1-\kappa \iota)\right)\right]$ and $b_{n}=\alpha_{n} \kappa \iota\left(1-\beta_{n}\right) \| x^{*}-$ $y^{*}||$. By the condition (iii), we get

$$
1>\kappa \iota, 1>\lambda_{n}>\alpha_{n}(1-\kappa \iota), \quad \forall n \in Z_{+} .
$$

In addition, from the conditions (i) and (ii), it yields that $b_{n}=0\left(\lambda_{n}\right)$ and

$$
\sum_{n=0}^{\infty} \lambda_{n}=\infty .
$$

Therefore, by Lemma 2.2, we obtain

$$
\lim _{n \rightarrow \infty} a_{n}=0,
$$

that is, $x_{n} \rightarrow x^{*}$ as $n \rightarrow \infty$. Again from $\lim _{n \rightarrow \infty} \beta_{n}=1$ and (4.6), one concludes

$$
\lim _{n \rightarrow \infty}\left\|y_{n}-y^{*}\right\|=0,
$$

i.e., $y_{n} \rightarrow y^{*}$ as $n \rightarrow \infty$. Thus $\left(x_{n}, y_{n}\right)$ converges strongly to $\left(x^{*}, y^{*}\right)$. This completes the proof. $\square$

Theorem 4.2. Let $E$ be a real $q$-uniformly smooth Banach space, and $A_{1}, A_{2}, M_{1}$ and $M_{2}$ be the same as in Theorem 3.5, and let $T$ be a $\kappa$-Lipschitz continuous mapping. Assume that $\Omega \cap F(T) \neq \varnothing,\left\{\alpha_{n}\right\}$ and $\left\{\beta_{n}\right\}$ are sequences in $[0,1]$ and satisfy the 
following conditions:

(i) $\sum_{i=0}^{\infty} \alpha_{n}=\infty$;

(ii) $\lim _{n \rightarrow \infty} \beta_{n}=1$;

(iii) $0<\kappa \sqrt[q]{1-q \rho_{i} \alpha_{i}+c_{q} \rho_{i}^{q} \tau_{i}^{q}}<1, i=1,2$.

Then the sequences $\left\{x_{n}\right\}$ and $\left\{y_{n}\right\}$ generated by Algorithm 4.1 converge strongly to $x^{*}$ and $y^{*}$, respectively, such that $\left(x^{*}, y^{*}\right) \in \Omega$ and $\left\{x^{*}, y^{*}\right\} \subset F(T)$.

Proof. The proof is similar to the proof of Theorem 4.1 and so the proof is omitted. This completes the proof. $\square$

Theorem 4.3. Let $E$ be a real $q$-uniformly smooth Banach space, and $A_{1}, A_{2}, M_{1}$ and $M_{2}$ be the same as in Theorem 3.4, and let $T$ be a $\kappa$-Lipschitz continuous mapping. Assume that $\Omega \cap F(T) \neq \varnothing,\left\{\alpha_{n}\right\}$ is a sequence in $(0,1]$ and satisfy the following conditions:

(i) $\sum_{i=0}^{\infty} \alpha_{n}=\infty$;

(ii) $0<\kappa \sqrt[q]{1-q \rho_{i} \nu_{i}+q \rho_{i} \mu_{i} \tau_{i}^{q}+c_{q} \rho_{i}^{q} \tau_{i}^{q}}<1, i=1,2$.

Then the sequences $\left\{x_{n}\right\}$ and $\left\{y_{n}\right\}$ generated by Algorithm 4.2 converge strongly to $x^{*}$ and $y^{*}$, respectively, such that $\left(x^{*}, y^{*}\right) \in \Omega$ and $\left\{x^{*}, y^{*}\right\} \subset F(T)$. Furthermore, sequences $\left\{x_{n}\right\}$ and $\left\{y_{n}\right\}$ are at least linear convergence.

Proof. From the proof of Theorem 4.1, it is easy to know that the sequences $\left\{x_{n}\right\}$ and $\left\{y_{n}\right\}$ generated by Algorithm 4.2 converge strongly to $x^{*}$ and $y^{*}$, respectively, such that $\left(x^{*}, y^{*}\right) \in \Omega$ and $\left\{x^{*}, y^{*}\right\} \subset F(T)$, and so,

$$
\begin{aligned}
& \left\|x_{n+1}-x^{*}\right\| \leq\left[1-\alpha_{n}\left(1-(\kappa \iota)^{2}\right)\right]\left\|x_{n}-x^{*}\right\|, \\
& \left\|y_{n}-\gamma^{*}\right\| \leq \kappa \sqrt[q]{1-q \rho_{2} v_{2}+q \rho_{2} \mu_{2} \tau_{2}^{q}+c_{q} \rho_{2}^{q} \tau_{2}^{q}}\left\|x_{n}-x^{*}\right\| .
\end{aligned}
$$

Since $\left\{\alpha_{n}\right\}$ is a sequence in $(0,1]$, we obtain, from (4.5),

$$
0<1-\alpha_{n}\left(1-(\kappa \iota)^{2}\right)<1
$$

and so,

$$
0<\kappa \sqrt[q]{1-q \rho_{2} v_{2}+q \rho_{2} \mu_{2} \tau_{2}^{q}+c_{q} \rho_{2}^{q} \tau_{2}^{q}}<1 .
$$

Therefore, from (4.7)-(4.10), it implies that sequences $\left\{x_{n}\right\}$ and $\left\{y_{n}\right\}$ are at least linear convergence. This completes the proof.

Theorem 4.4. Let $E$ be a real $q$-uniformly smooth Banach space, and $A_{1}, A_{2}, M_{1}$ and $M_{2}$ be the same as in Theorem 3.5, and let $T$ be a $\kappa$-Lipschitz continuous mapping. Assume that $\Omega \cap F(T) \neq \varnothing,\left\{\alpha_{n}\right\}$ is a sequence in $(0,1]$ and satisfy the following conditions:

(i) $\sum_{i=0}^{\infty} \alpha_{n}=\infty$;

(ii) $\lim _{n \rightarrow \infty} \beta_{n}=1$; 
(iii) $0<\kappa \sqrt[q]{1-q \rho_{i} \alpha_{i}+c_{q} \rho_{i}^{q} \tau_{i}^{q}}<1, i=1,2$.

Then the sequences $\left\{x_{n}\right\}$ and $\left\{y_{n}\right\}$ generated by Algorithm 4.2 converge strongly to $x^{*}$ and $y^{*}$, respectively, such that $\left(x^{*}, y^{*}\right) \in \Omega$ and $\left\{x^{*}, y^{*}\right\} \subset F(T)$. Furthermore, sequences $\left\{x_{n}\right\}$ and $\left\{y_{n}\right\}$ are at least linear convergence.

Proof. In a way similar to the proof of Theorem 4.2, with suitable modifications, we can obtain that the conclusion of Theorem 4.4 holds. This completes the proof. $\square$

Remark 4.2. Theorem 4.1 generalizes and improves the main result in [18].

\section{Abbreviation}

(SGQVI): system of generalized quasivariational inclusion.

\section{Acknowledgements}

The authors would like to thank two anonymous referees for their valuable comments and suggestions, which led to an improved presentation of the results, and grateful to Professor Siegfried Carl as the Editor of our paper. This work was supported by the Natural Science Foundation of China (Nos. 71171150,70771080,60804065), the Academic Award for Excellent Ph.D. Candidates Funded by Wuhan University and the Fundamental Research Fund for the Central Universities.

\section{Author details}

${ }^{1}$ School of Mathematics and Statistics, Wuhan University, Wuhan, Hubei 430072, PR China ${ }^{2}$ School of Mathematics and information, China West Normal University, Nanchong, Sichuan 637002, PR China

\section{Authors' contributions}

$J C$ carried out the (SGQVI) studies, participated in the sequence alignment and drafted the manuscript. ZW participated in the sequence alignment. All authors read and approved the final manuscript.

\section{Competing interests}

The authors declare that they have no competing interests.

Received: 23 March 2011 Accepted: 5 September 2011 Published: 5 September 2011

\section{References}

1. Stampacchia, G: Forms bilineaires coercivities sur les ensembles convexes. CR Acad Sci Paris. 258, 4413-4416 (1964)

2. Ansari, QH, Schaible, S, Yao, JC: System of vector equilibrium problems and its applications. J Optim Theory Appl. 107(3), 547-557 (2000). doi, 10.1023/A:1026495115191

3. Agarwal, RT, Huang, NJ, Tan, MY: Sensitivity analysis for a new system of generalized non-linear mixed quasi-variational inclusions. Appl Math Lett. 17(3), 345-352 (2004). doi, 10.1016/S0893-9659(04)90073-0

4. Chen, JW, Cho, YJ, Kim, JK, Li, J: Multiobjective optimization problems with modified objective functions and cone constraints and applications. J Glob Optim. 49(1), 137-147 (2011). doi, 10.1007/s10898-010-9539-3

5. Chen, JW, Zou, YZ: Existence of solutions of F-implicit variational inequality problems with extended projection operators. Acta Math Sin Chin Ser. 53(2), 375-384 (2010)

6. Verma, RU: Projection methods, algorithms, and a new system of nonlinear variational inequalities. Comput Math Appl. 41(7-8), 1025-1031 (2001). doi, 10.1016/50898-1221(00)00336-9

7. Kim, JK, Kim, DS: A new system of generalized nonlinear mixed variational inequalities in Hilbert spaces. J Convex Anal. 11(1), 235-243 (2004)

8. Cho, YJ, Fang, YP, Huang, NJ, Hwang, NJ: Algorithms for systems of nonlinear variational inequalities. J Korean Math Soc. 41(3), 203-210 (2004)

9. Peng, JW, Zhu, DL: Existence of solutions and convergence of iterative algorithms for a system of generalized nonlinear mixed quasi-variational inclusions. Comput Math Appl. 53(5), 693-705 (2007). doi, 10.1016/j.camwa.2006.07.005

10. Huang, NJ, Fang, YP: Generalized m-accretive mappings in Banach spaces. J Sichuan Univ (Natural Science Edition). 38(4), 591-592 (2001)

11. Huang, NJ: Random general set-valued strongly nonlinear quasi-variational inequalities. J Sichuan Univ (Natural Science Edition). 31, 420-425 (1994)

12. Huang, NJ: Generalized nonlinear variational inclusions with noncompact valued mappings. Appl Math Lett. 9(3), 25-29 (1996). doi, 10.1016/0893-9659(96)00026-2

13. Huang, NJ, Cho, YJ: Random completely generalized set-valued implicit quasivariational in-equalities. Positive. 3 , 201-213 (1999). doi:10.1023/A:1009784323320

14. Pang, JS: Asymmetric variational inequality problems over product sets: applications and iterative methods. Math Programming. 31(2), 206-219 (1985). doi, 10.1007/BF02591749

15. Qin, X, Chang, SS, Cho, YJ, Kang, SM: Approximation of solutions to a system of variational inclusions in Banach spaces. J Inequality Appl 2010, 16 (2010). Article ID916806

16. Kamraksa, U, Wangkeeree, R: A general iterative process for solving a system of variational inclusions in Banach spaces. J Inequality Appl 2010, 24 (2010). Article ID190126 
17. Wangkeeree, R, Kamraksa, U: An iterative approximation method for solving a general system of variational inequality problems and mixed equilibrium problems. Nonlinear Anal. 3, 615-630 (2009)

18. Petrot, $\mathrm{N}$ : A resolvent operator technique for approximate solving of generalized system mixed variational inequality and fixed point problems. Appl Math Lett. 23, 440-445 (2010). doi:10.1016/j.aml.2009.12.001

19. Zhao, Y, Xia, Z, Pang, L, Zhang, L: Existence of solutions and algorithm for a system of variational inequalities. Fixed Point Theory Appl 2010, 11 (2010). Article ID 182539

20. Qin, X, Cho, SY, Kang, SM: Convergence of an iterative algorithm for systems of variational inequalities and nonexpansive mappings with applications. J Comput Appl Math. 233, 231-240 (2009). doi:10.1016/j.cam.2009.07.018

21. Deimling, K: Nonlinear Functional Analysis. Springer, Berlin (1985)

22. He, Z, Gu, F: Generalized system for relaxed cocoercive mixed variational inequalities in Hilbert spaces. Appl Math Comput. 214, 26-30 (2009). doi:10.1016/j.amc.2009.03.056

23. Chang, SS, Joseph Lee, HW, Chan, CK: Generalized system for relaxed cocoercive variational inequalities in Hilbert spaces. Appl Math Lett. 20, 329-334 (2007). doi:10.1016/j.aml.2006.04.017

24. Verma, RU: Approximation-solvability of a class of A-monotone variational inclusion problems. J Korean Soc Ind Appl Math. 8(1), 55-66 (2004)

25. Verma, RU: A-monotonicity and applications to nonlinear variational inclusion problems. J Appl Math Stoch Anal. 2, 193-195 (2004)

26. Verma, RU: General over-relaxed proximal point algorithm involving A-maximal relaxed monotone mapping with applications. Nonlinear Anal. 71(12), 1461-1472 (2009). doi, 10.1016/j.na.2009.01.184

27. Aoyama, K, Kimura, Y, Takahashi, W, Toyoda, M: On a strong nonexpansive sequence in Hilbert spaces. J Nonlinear Convex Anal. 8(3), 471-489 (2007)

28. Weng, XL: Fixed point iteration for local strictly pseudocontractive mappings. Proc Am Math Soc. 113, 727-731 (1991). doi:10.1090/S0002-9939-1991-1086345-8

29. Xu, HK: Inequalities in Banach spaces with applications. Nonlinear Anal. 16, 1127-1138 (1991). doi:10.1016/0362-546X(91) $90200-K$

doi:10.1186/1029-242X-2011-49

Cite this article as: Chen and Wan: Existence of solutions and convergence analysis for a system of quasivariational inclusions in Banach spaces. Journal of Inequalities and Applications 2011 2011:49.

\section{Submit your manuscript to a SpringerOpen ${ }^{\odot}$ journal and benefit from:}

Convenient online submission

- Rigorous peer review

- Immediate publication on acceptance

- Open access: articles freely available online

- High visibility within the field

- Retaining the copyright to your article

Submit your next manuscript at $\gg$ springeropen.com 